\title{
Computer aided design of stable and efficient OLEDs
}

Cite as: J. Appl. Phys. 128, 160901 (2020); https://doi.org/10.1063/5.0022870

Submitted: 24 July 2020 . Accepted: 08 October 2020 . Published Online: 22 October 2020

Leanne Paterson, Falk May, and (D) Denis Andrienko
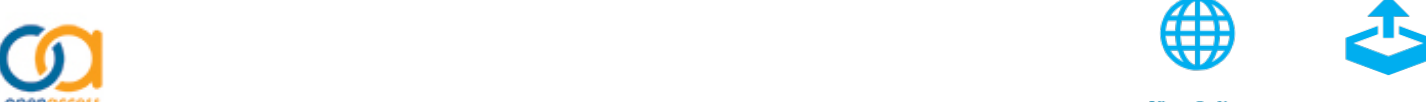

View Online

\section{ARTICLES YOU MAY BE INTERESTED IN}

Frontiers in atomistic simulations of high entropy alloys

Journal of Applied Physics 128, 150901 (2020); https://doi.org/10.1063/5.0025310

Introduction to spin wave computing

Journal of Applied Physics 128, 161101 (2020); https://doi.org/10.1063/5.0019328

Ultra-compact mechanical antennas

Applied Physics Letters 117, 170501 (2020); https://doi.org/10.1063/5.0025362

\section{Meet the Next Generation of Quantum Analyzers And Join the Launch Event on November 17th}

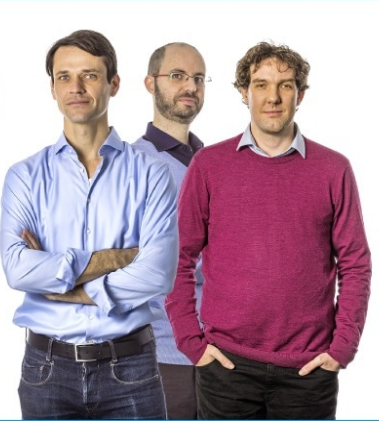

\section{Register now}

Zurich Instruments 


\title{
Computer aided design of stable and efficient OLEDs
}

\author{
Cite as: J. Appl. Phys. 128, 160901 (2020); doi: 10.1063/5.0022870 \\ Submitted: 24 July 2020 - Accepted: 8 October 2020 . \\ Published Online: 22 October 2020
}

Leanne Paterson, ${ }^{7}$ Falk May, ${ }^{2}$ and Denis Andrienko ${ }^{1, a)}$ (iD

AFFILIATIONS

${ }^{1}$ Max Planck Institute for Polymer Research, Ackermannweg 10, 55128 Mainz, Germany

${ }^{2}$ Merck KGaA, 64293 Darmstadt, Germany

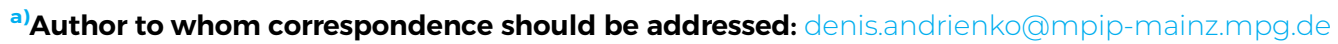

\begin{abstract}
Organic light emitting diodes (OLEDs) offer a unique alternative to traditional display technologies. Tailored device architecture can offer properties such as flexibility and transparency, presenting unparalleled application possibilities. Commercial advancement of OLEDs is highly anticipated, and continued research is vital for improving device efficiency and lifetime. The performance of an OLED relies on an intricate balance between stability, efficiency, operational driving voltage, and color coordinates, with the aim of optimizing these parameters by employing an appropriate material design. Multiscale simulation techniques can aid with the rational design of these materials, in order to overcome existing shortcomings. For example, extensive research has focused on the emissive layer and the obstacles surrounding blue OLEDs, in particular, the trade-off between stability and efficiency, while preserving blue emission. More generally, due to the vast number of contending organic materials and with experimental pre-screening being notoriously time-consuming, a complementary in silico approach can be considerably beneficial. The ultimate goal of simulations is the prediction of device properties from chemical composition, prior to synthesis. However, various challenges must be overcome to bring this to a realization, some of which are discussed in this Perspective. Computer aided design is becoming an essential component for future OLED developments, and with the field shifting toward machine learning based approaches, in silico pre-screening is the future of material design.
\end{abstract}

(C) 2020 Author(s). All article content, except where otherwise noted, is licensed under a Creative Commons Attribution (CC BY) license (http:// creativecommons.org/licenses/by/4.0/). https://doi.org/10.1063/5.0022870

\section{INTRODUCTION}

Organic light emitting diodes (OLEDs) have attracted much interest in recent years, from the pivotal discovery of electroluminescent properties in certain organic materials ${ }^{1-3}$ and the fabrication of the first device ${ }^{4}$ to applications in present day technologies. With display applications being an essential component of many modern electronic devices, the unique properties of OLEDs have propelled them into the industry. Every day, functionality, reliability, and efficiency drive electronic devices forward, and with these devices advancing at an unprecedented rate, it is imperative that the technology behind them follows the same trend. The mechanically flexible, transparent, and lightweight properties of OLEDs create a whole host of new technologies and applications in this rapidly expanding market. Therefore, the advancement of these devices is highly anticipated. In comparison to rigid inorganic LEDs, OLEDs offer a unique flexible substitute, with possibilities including curved, foldable, and wearable displays. Additionally, as they do not require a backlight, a better contrast ratio and an overall improved image quality are achieved, compared with the LCD technology. OLEDs can already be found in a host of commercial applications from the automotive industry to wearable and mobile devices, such as smartphones and watches. While OLEDs offer an enticing substitute, they remain in their infancy with respect to their inorganic counterparts, so continued research is essential in improving device efficiency and lifetime.

So, what is an OLED? As a sub-category of organic semiconductors, OLEDs are carbon-based compounds with structures tailored for photo- or electro-luminescence. They utilize a thin layer of a polymeric or small molecule based organic materials to achieve a desirable wavelength of emission. A typical (small molecule) multilayer OLED structure sandwiched between two electrodes is illustrated in Fig. 1, showing each layer with the corresponding function. Upon the application of an external potential, electrons and holes are injected from the cathode and anode, respectively. Electron/hole 
(a)

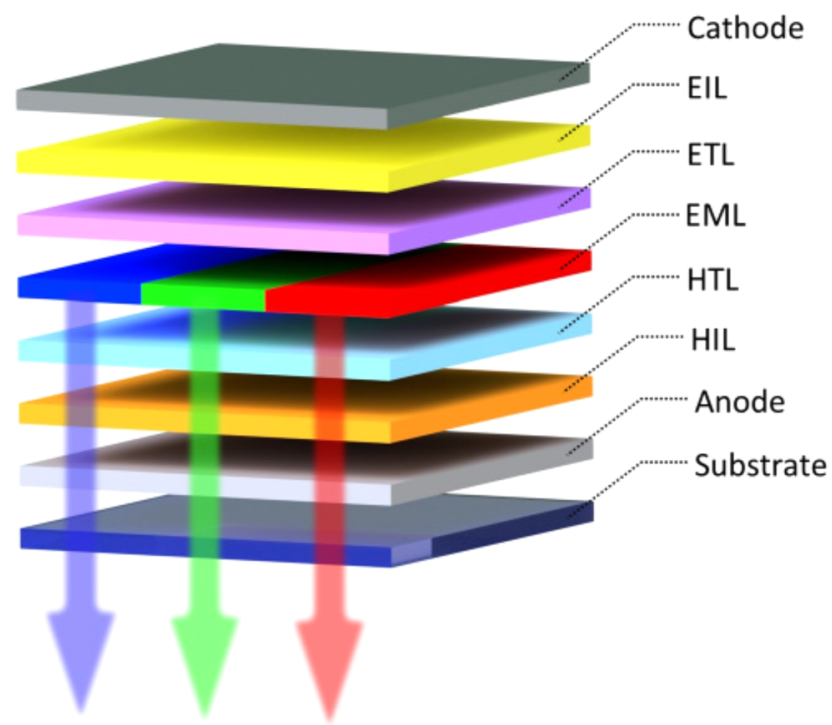

(b)

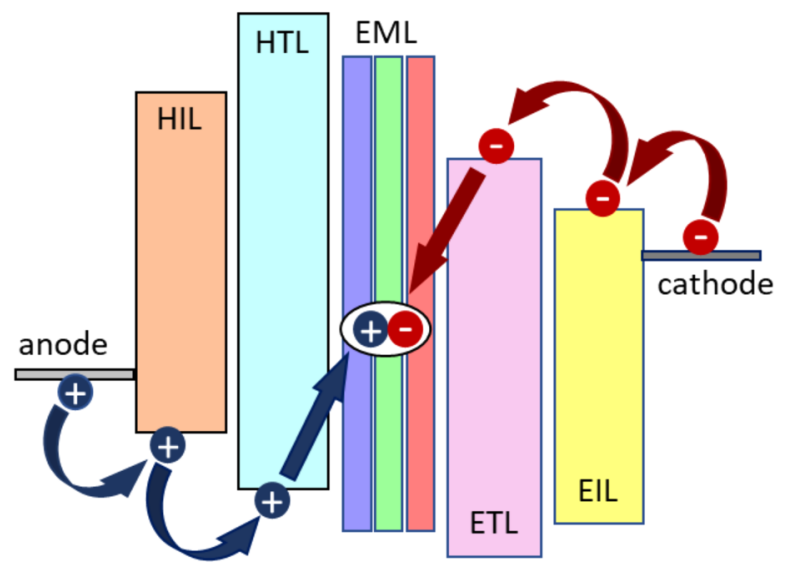

FIG. 1. (a) Basic OLED structure: electrons are injected at the cathode to the electron injection layer (EIL) and transported via the electron transport layer (ETL); holes are injected from the anode to the hole injection layer (HIL) and transported through the hole transport layer (HTL). Both then combine in the emissive layer (EML) to form an exciton and emit a photon of specific wavelength. (b) Schematic representation of the flow of electrons and holes from electrodes to the EML.

injection and transport layers then facilitate the movement of the charge carriers to the emissive layer (EML), where they recombine to form excitons, and consequently, a photon of the desirable wavelength is emitted. A blocking layer, in combination with, or additional to the transport layer for each carrier can also be utilized to assist in the accumulation of carriers in the emissive layer by preventing electron transport to the anode and hole transport to the cathode.

Various factors affect the performance of an OLED, and typically, there is never an "ideal" solution to obtain an optimized device in all relevant parameters. A compromise between stability, efficiency, operational driving voltage, and color coordinate is inevitable. However, with an appropriate material design, it is possible to achieve devices that balance these individual limitations. This has been demonstrated for an emissive layer, with the novel concept of unicolored phosphor-sensitized fluorescence (UPSF), where a phosphorescent donor and fluorescent acceptor achieve a trade-off between stability and efficiency, while preserving the sky-blue emission color. Maximizing the device performance previously focused solely on improving the stability of the organic materials used. However, due to the advent of the new $5 \mathrm{G}$ standard for mobile applications, the focus has shifted toward increasing the efficiency and reducing the driving voltage of each layer. The functionality of the layer, which is determined by molecular architecture, electronic properties, and charge carrier mobilities, relies on the choice of organic materials. The individual choice of each layer and each emitter is crucial in enhancing the OLED in its entirety. Charge transporting and emissive layers have to be designed in such a way as to maximize their function and stability. By utilizing an electron and hole injection and transport layer, the device performance has been dramatically improved already., ${ }^{2,-9}$ Therefore, tremendous research efforts have involved tailoring of electron injection and transport layers, hole injection and transport layers, and the various emitters for the emissive layer, as well as potential degradation mechanisms ${ }^{10-13}$ and reactions within the OLED device, ${ }^{13}$ to target stability improvements, all of which are necessary in the path toward high performing and long-lasting OLEDs.

Considering the individual emitters, at present, there exist stable and efficient triplet emitters for red, ${ }^{14-23}$ green, ${ }^{24-33}$ and yellow (for use in white OLED-TV stacks), with blue falling short. Blue emitters continue to be problematic and many ongoing research efforts target this specifically. ${ }^{34}$ The focus is most often on the emissive paths, be that fluorescence, ${ }^{35}$ phosphorescence, ${ }^{36-38}$ thermally activated delayed fluorescence (TADF), ${ }^{39-44}$ or sensitizing approaches. ${ }^{5,6}$ This ranges from understanding degradation mechanisms in phosphorescent emitters ${ }^{45-48}$ to utilizing combinations of emitters to overcome individual limitations. ${ }^{5,49,50}$ Although blue emitters are most notably challenging, overall increased stability and efficiency of all emitters and the device as a whole remain challenges for commercial OLED development.

With many potential candidates for each emitter and many potential emissive paths, it is crucial that simulations provide information and a deeper insight, which otherwise would not be accessible. Simulations can be used to study each of the OLED layers and the contending organic materials, as well as possible degradation mechanisms, all toward a better understanding of the internal processes and highlighting key areas of improvement. There are well established methods for helping to optimize the stacked OLED architecture, by modeling light outcoupling and the balancing of hole and electron transport. $^{51,52}$ The next step is to incorporate molecular details and achieve a mechanism of evaluating an OLED chemical design. ${ }^{53,54}$ 
The goal of simulations is to predict physical properties from chemical composition, also known as the forward problem; this would allow for pre-screening and an overall better insight. Simulations can not only be advantageous but also are an essential tool in the design of future OLEDs. This extraction of properties from the molecular structure alone is, of course, non-trivial, and computational pre-screening is not yet accurate enough for this task exclusively. ${ }^{55}$ However, a collective experimental and in silico approach can be beneficial due to the fact that there are a vast number of potential candidates and experimental pre-screening is notoriously time-consuming.

This Perspective will discuss the current shortcomings of OLEDs, in the search for increased device lifetime and efficiency, and how multiscale simulations can assist OLED design, including predictions of electronic properties, such as ionization energy, electron affinity, and electron and hole mobility. Additionally, it gives further insight into internal processes and highlights potential areas for improvement in existing devices. The prediction of microscopic device properties will be discussed, including the steps that would be required for the extraction of valuable properties from the molecular structure alone. We will then look at the possible direction the field may take, considering atomistic scale machine learning, with machine learning based approaches gaining significant momentum in most computational areas.

\section{IMPROVING DEVICE PERFORMANCE}

The path of exciton formation and decay resulting in photon emission, as well as the emission color, depends on the organic molecules and molecular packing. It is crucial to utilize the individual strengths of organic materials for their designated task. As a result, extensive research efforts have focused on each of the individual layers and the organic constituents. The "one by one" layer approach is more practical, as chemical properties of the molecules can be tuned for specific OLED characteristics. Listed below is a short introduction to each of the OLED layers, with an example of how the specific challenges are addressed, paying particular attention to the emissive layer and the challenges surrounding blue emitters.

\section{Cathode and electron injection layer (EIL)}

The injection of electrons into the organic layers has a significant impact on the efficiency of the OLED. Lowering the energetic barrier between the cathode and the lowest unoccupied molecular orbital (LUMO) of the adjacent organic layer facilitates the injection of electrons. Low work function metal alloy cathodes, such as $\mathrm{Mg}: \mathrm{Al},{ }^{4}$ are susceptible to atmospheric conditions. ${ }^{56}$ Therefore, for increased stability, cathode bilayer structures, such as $\mathrm{MgAg} / \mathrm{Ag}^{14,57}$ and $\mathrm{LiF} / \mathrm{Al},{ }^{56,58,59}$ have been frequently used. Numerous other studies have investigated n-type metal oxide semiconductors ${ }^{60}$ and alkali metal containing interlayers. ${ }^{61,62}$

\section{Electron transport layer (ETL)}

The electron transport layer facilitates the movement of electrons toward the emissive layer and, therefore, is a vital component of the device, confirmed by the extensive research on ETL materials. ${ }^{8,63}$ Since balancing electron and hole injection and transport to the emissive layer is crucial for OLED performance, the challenge revolves around finding suitable and stable materials with high charge carrier mobilities. Within electron or hole transporting materials, the presence of energy traps can have a detrimental impact on the charge carrier mobilities. For large energy gap materials, this is due to the fact that either the electron affinity or ionization energy lies in a trap region, such that unipolarity prevails. Specifically, when considering the ETL, a shallow electron affinity results in trap limited electron transport and low electron mobility. It has been shown that there is in fact an energy window, within which there lie materials for trap-free ambipolar transport, resulting in higher mobilities. ${ }^{64}$ This is an ionization energy below $6 \mathrm{eV}$ for hole transport and an electron affinity above $3.6 \mathrm{eV}$, such that for an appropriate material design, ideally, this energy window should be targeted. Additionally, for optimal emission, the ETL should block holes and excitons from escaping the emissive layer. Therefore, the ETL layer should have a small injection barrier for electrons from the EIL or cathode, a highest occupied molecular orbital (HOMO) level low enough to effectively block holes from the emissive layer, and a high triplet energy level for the case of triplet excitons (with high diffusion lengths). Considering one material among many various options, $\mathrm{Alq}_{3}$ has been extensively studied for electron transport (as well as hole transport and emissive layers), spanning from the first OLED fabrication. ${ }^{4}$ However, as it has a low triplet energy level, ${ }^{65}$ it cannot be used with an EML creating triplet excitons, unless coupled with an interlayer. For example, the use of a hole and exciton blocking layer, such as BCP in combination with $\mathrm{Alq}_{3}$, has been demonstrated to increase efficiency. ${ }^{14,15,24,57}$ A further option is improving the thermal stability of materials with high electron mobility, such as BPhen, demonstrated with the use of alkali metal n-dopants. ${ }^{66}$ Another alternative is the use of triazine based electron transporters, where substituents can be used to tune the electron mobility and LUMO energy for adjusted injection.

\section{Hole injection layer (HIL)}

Inserted between the typically used transparent indium-tin-oxide (ITO) anode and the hole transport layer (HTL), the hole injection layer eases hole migration at each of the interfaces. Materials for the HIL should have an ionization energy level situated between that of the preceding and succeeding layers. This is slightly easier for hole injection, in comparison to electron injection, due to the typically lower injection barrier. Nevertheless, it has been shown that the stepwise injection from anode to HIL to HTL can improve performance; such an instance has been shown with the use of an organic interlayer, ${ }^{67}$ such as MTDATA or 2-TNATA. ${ }^{68}$

\section{Hole transport layer (HTL)}

For a HTL to be effective, the material should fulfill certain properties. It should have good hole mobility and appropriate HOMO level to ensure a low energetic barrier from the HIL, for hole transport, and a suitable LUMO level to act as an electron blocking layer. Similar to the ETL, HTL materials also need sufficient triplet energies to confine the exciton within the EML. The most widely used HTL materials are arylamines, such as NPB, due to their high hole mobility and suitable HOMO levels. However, due to its 
low glass transition temperature, NPB itself has low thermal stability, which can lead to device degradation. The vast research in recent years ${ }^{69}$ has focused on finding more stable NPB derivatives or alternatives with similar or improved performance. ${ }^{70-72}$

\section{Emissive layer (EML)}

Red, green, and blue emitters are essential for full color displays. The goal is to achieve an emission layer of each color with high luminous properties, high efficiency, and high stability. Independent of the emission path, in order to maximize the outcoupling efficiency, the transition dipole moment of the emitter should be aligned horizontally with respect to the substrate plane. ${ }^{73}$ Also here, computer simulations of the evaporation process can help in predicting how emitter-host interactions can be employed in a rational compound design. ${ }^{74}$

Localized energy states, resulting in well-defined singlet and triplet spin states within the OLED, aid with the luminescent properties. ${ }^{75}$ The excitation of the molecule involves the promotion of an electron from the singlet ground state $\left(\mathrm{S}_{0}\right)$ to the first excited singlet state $\left(S_{1}\right)$ or the first triplet excited state $\left(T_{1}\right)$, as shown in Fig. 2. Excitation occurs with a $25 \%$ probability to the $S_{1}$ state, as there is only one possible configuration (maintaining antiparallel spin), or a $75 \%$ probability to the $T_{1}$ state, with three potential parallel spin combinations.

First generation fluorescent emitting OLEDs typically have high stability but low efficiency. This is due to an unfavorable spin transition between the singlet and triplet states, with rapid radiative $(\sim n s)$ decay only readily occurring from the $S_{1}$ state, resulting in around a $25 \%$ efficiency of the emitter. Increasing the overall efficiency in fluorescent emitters is possible using triplet-triplet annihilation, which is often facilitated by anthracene based hosts.

Second generation OLEDs or phosphorescent emitters, comprised of organometallic complexes, have high efficiency but lower stability. Here, radiative decay is possible from the $T_{1}$ state, resulting in slow $(\sim \mu \mathrm{s})$ phosphorescent emission. Additionally, the presence of a heavy metal atom, such as iridium or platinum, with an appropriate ligand design, results in spin-orbit coupling. This

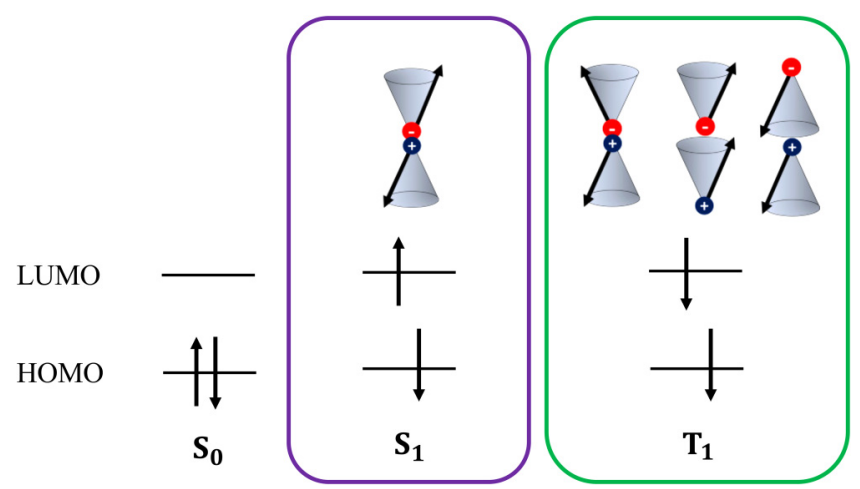

FIG. 2. Electronic configuration for the ground state $\left(\mathrm{S}_{0}\right)$, the first singlet excited state $\left(S_{1}\right)$, and the first triplet excited state $\left(T_{1}\right)$. The arrows represent the electron spin. allows for the transition between $S_{1}$ and $T_{1}$ states, known as intersystem crossing (ISC), which significantly increases the efficiency. The downfall being that these heavy metal complexes are particularly susceptible to environmental factors, which can lead to device degradation. The phosphorescent long-lived excited triplet state, typically in the order of microseconds (compared to nanoseconds of the fluorescent $S_{1}$ state), also leads to degradation, and as such, these emitters have low stability.

Third generation OLEDs that use TADF emitters harvest the triplet excitons lost in conventional fluorescent emitters by making use of a thermally activated process called reverse inter-system crossing (RISC). Choosing a sufficiently low energy gap between the $T_{1}$ and $S_{1}$ levels allows for RISC and can result in highly efficient and stable emitters.

Finally, a sensitizing approach is possible, combining a donor and acceptor molecule, with the aim of overcoming the individual limitations of both emitters. One such example is the use of a phosphorescent donor and a fluorescent acceptor. We will discuss the application of this mechanism, with a unicolored phosphorsensitized fluorescence (UPSF) approach, for the realization of a sky-blue OLED. The various emissive paths are shown in Fig. 3, outlining the energy levels and radiative or non-radiative (NR) decay for each type of emitters, or combinations. In its entirety, the emissive layer consists of the chosen type(s) of emitter(s), typically dispersed within a charge transporting host material to increase efficiency. Usually, this combats adverse factors, such as triplettriplet annihilation in phosphorescent emitters, for example.

Currently, there are stable and efficient red and green emitters available, with extensive research focusing on both. Due to high efficiency, phosphorescent emitters have dominated the field, in the search for red ${ }^{14-20}$ and green emitters. ${ }^{24-28}$ Various charge transporting host materials have been the subject of investigation to understand the relationship between host and device performance, targeting hosts to combat potential efficiency losses. For a similar purpose, double emissive layers have also been studied. ${ }^{76,77}$ Additionally, $\operatorname{red}^{21-23}$ and green ${ }^{29-33}$ TADF emitters have been shown to be efficient, low cost alternatives to the expensive precious heavy metals found in phosphorescent emitters.

As previously stated, blue emitters are particularly problematic due to higher triplet energy and long triplet lifetimes, leading to the lowest stability. This remains one of the largest hurdles for commercial OLED applications to date. Immense efforts have centered around finding solutions to limit device degradation in phosphorescent emitters or utilizing hybrid emissive technology in an attempt to overcome it. The short operational lifetime of blue phosphorescent emitters has resulted in studies of degradation mechanisms. ${ }^{45-48}$ Once understood, these limitations can be addressed, potentially increasing stability. Specifically, this has included targeting adverse factors such as (1) chemical degradation, ${ }^{45}$ (2) triplet-polaron quenching, ${ }^{38,46,47,78,79}$ and (3) triplet-triplet annihilation. ${ }^{80-82}$ Additionally, simulations have been used to investigate host materials for efficient charge transport within blue phosphorescent emitters, ${ }^{83}$ providing a link between the electronic structure and molecular packing, to the rational design of effective host materials with high charge carrier mobilities. Blue TADF emitters ${ }^{39-44}$ and combinations of TADF with conventional fluorescent ${ }^{84,85}$ or phosphorescent emitters $^{84,86}$ have also been investigated. This includes the impact of 


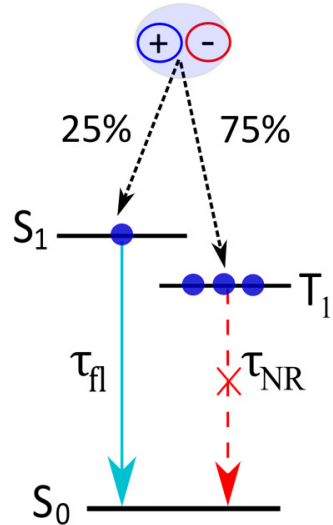

Fluorescence

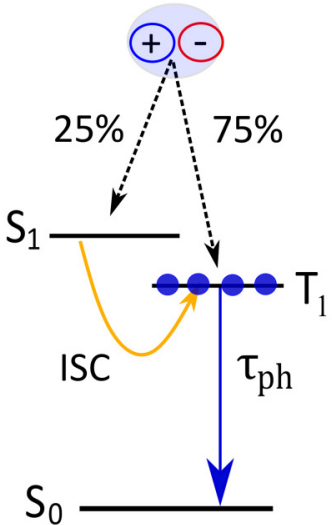

Phosphorescence

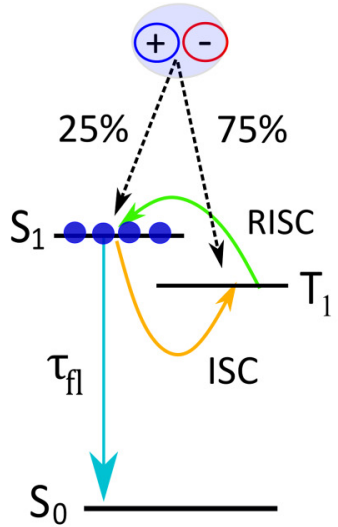

TADF

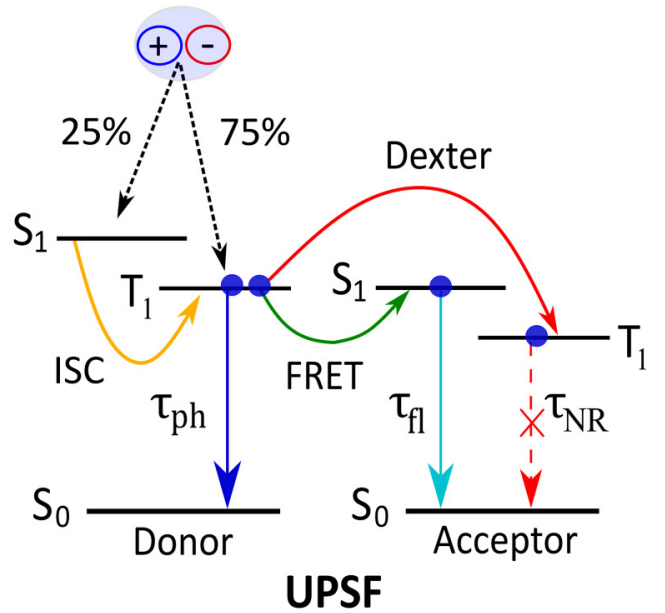

FIG. 3. Various emissive paths: fluorescence (fl) with $25 \%$ efficiency only emits from the first singlet state. Phosphorescence (Ph) with an intersystem crossing (ISC) allows for complete emission from the first triplet state. Thermally activated delayed fluorescence (TADF) makes use of the reverse intersystem crossing (RISC) to emit from the first singlet state more efficiently. Unicolored phosphor-sensitized fluorescence (UPSF) with a phosphorescent donor emitting from the first triplet state and energy transfer occurring via FRET or Dexter to the singlet or triplet of a fluorescent acceptor. Radiative decay can occur from the singlet of the acceptor but the acceptor triplet is a non-radiative (NR) decay pathway.

emitter-host interaction $^{42,43}$ and methods of lowering the singlet-triplet energy gap, ${ }^{44}$ potentially enhancing the performance. However, at present, with triplet lifetimes similar to that of phosphorescent emitters, the low stability problem remains for blue TADF based systems.

A phosphor-sensitized fluorescence approach ${ }^{49,87-90}$ offers an alternative to conventional phosphorescent and TADF emitters for various emitting wavelengths, in an effort to overcome their individual shortcomings, by coupling both fluorescent and phosphorescent emitters. By utilizing a phosphorescent donor and a fluorescent acceptor, distributed within a host, it is possible to obtain a dual emitting system that is both stable and efficient. A unicolored phosphor-sensitized fluorescence (UPSF) approach, with matching donor and acceptor emission color, was recently proposed for blue OLEDs. ${ }^{5,6}$ The energy transfer processes and radiative decay paths of the phosphorescent donor and fluorescent acceptor, for the UPSF system, are illustrated in Fig. 3. The phosphorescent donor can (1) emit from the $T_{1}$ state, or (2) transfer energy to the $S_{1}$ state of fluorescent acceptor or (3) the acceptor $T_{1}$ state. If the donor and acceptor molecules are adequately spaced $(>1 \mathrm{~nm})$, there will be a Förster resonance energy transfer (FRET) from the donor $T_{1}$ state to the acceptor $S_{1}$ state, resulting in fluorescence emission from the acceptor. On the other hand, if the molecules are closer in distance, a Dexter energy transfer will prevail, between the donor $T_{1}$ state the acceptor $T_{1}$ state, resulting in a loss of efficiency as this is a non-radiative state and no RISC is present. Experimentally, it was shown that by increasing the concentration of fluorescent acceptor molecules, the stability was increased and made apparent with a threefold increase in device lifetime. However, as a result of the probability of the Dexter energy transfer also increasing, the efficiency of the device decreases with increasing acceptor concentration, shown with a reduction in the photoluminescence quantum yield (PLQY). Therefore, it was shown that the UPSF OLED has to be designed to optimize the acceptor concentration for increased stability and operation lifetime, and target donor-acceptor combinations with slow Dexter and fast FRET rates for increased efficiency.

Our recently published complementary simulation study for this blue UPSF OLED approach ${ }^{91}$ demonstrates an essential computational component of such OLED designs. By providing a deeper insight for better understanding and expanding on the scope of experiment to look at the limitations of the concept, as well as key areas of potential improvement, computational input can be vital. We developed a multiscale model, as shown in Fig. 4, to investigate the UPSF OLED and highlight any inherent limitations of the concept. Additionally, as the simulations are based on the experimentally achieved results, it is possible to do so without explicit consideration of chemical design, starting with atomistic morphologies of the UPSF systems and parametrizing the rates (from experimental data) of all of the essential processes involved to then solving the respective master equation with the use of a kinetic Monte Carlo (KMC) algorithm.

First, it became apparent that an additional mode of energy transfer was missing from the original description and that transfer between donor molecules was also essential. This was taking place between the triplets of the respective donor molecules, as a donor to donor Dexter energy transfer, which was not obvious from experimental results. But, it was a mechanism which facilitated increased energy transfer between the donor and acceptor by allowing for a step-wise energy transfer process. Second, we could expand on the experimental results by studying increased acceptor concentrations in order to understand any limitation of the UPSF concept. A level of saturation was observed in terms of the number of donor to acceptor energy transfer processes, such that higher 


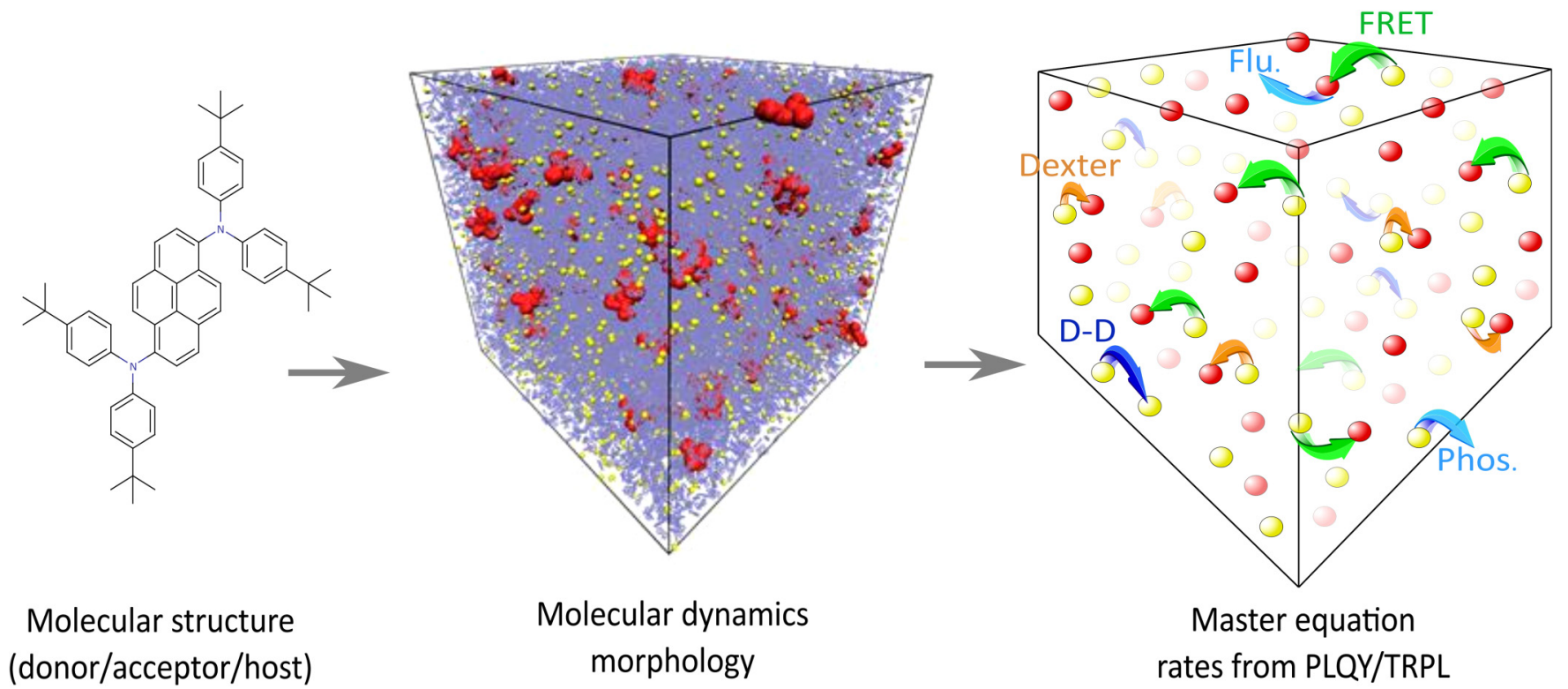

FIG. 4. Simulation workflow: initial molecular structures are used to construct a morphology for molecular dynamics simulations followed by rate parameterization from experimental data and then KMC to randomly propagate the system through time, providing OLED properties such as photoluminescence quantum yield (PLQY) and radiative decay times and plots such as time resolved photoluminescence (TRPL).

concentration of acceptor did not result in a higher number of FRET/Dexter events. Although the saturation of Dexter events may seem positive, it ultimately means that the UPSF OLED has an upper limit to the lifetime. When considering acceptor concentration to increase, less energy transfer from the donor to acceptor results in increased (long-lived) donor phosphorescent emission. Finally, we were able to demonstrate the potential of an ideal UPSF OLED design with Dexter energy transfer suppression and/or an increase of the radius in which FRET could occur. It was evident that the FRET radius had a significant impact on the OLED performance, exceeding the improvement from Dexter suppression alone. However, the chemical design of these materials, in particular, tuning of the FRET radius, is experimentally challenging, as it can easily lead to a compromise between efficiency, stability, and emission color. Therefore, we show that by combining experimental and computational results, it is possible to gain a better understanding of such a novel concept while highlighting key areas for further device improvements.

By addressing the role of exciton decay time in the UPSF system, we present one example of how simulations can address questions surrounding device stability. However, for a long time, the aspect of OLED stability has been underrepresented in academic research, partially because of a lack of access to highly purified materials or insufficient control over device fabrication methods. This problem can be overcome by collaboration between academia and industry such that nowadays stability limitations ranging from chemical ${ }^{92}$ to morphological changes ${ }^{93}$ in each of the OLED layers can be addressed. Computationally, it is possible to study chemical stability in terms of single-molecule properties, like bond-dissociation energies ${ }^{94}$ or more complex molecular degradation scenarios involving polarons and excitons. ${ }^{46}$ Simulations on the device level have also shown that the role of exciton-exciton or exciton-polaron interaction can play a substantial part in OLED stability. ${ }^{51}$ Therefore, being able to correctly describe the photophysics is a crucial part in understanding OLED stability limitations. This can be described as a multi-scale problem, including the single-molecule level, e.g., understanding fast intersystem crossing in TADF emitters, ${ }^{95}$ or on larger scales, e.g., triplet diffusion in the emissive layer ${ }^{96}$ or exciton-dynamics coupled to charge dynamics on the full device level. ${ }^{97}$

\section{OLED MATERIAL LIBRARY}

As shown for the UPSF OLED, an initial computational approach is to construct a model from the available experimental data, thus providing deeper insight. However, the ultimate goal of OLED simulations is gaining extensive understanding between molecular structure and physical properties, ideally before synthesis, due to the vast number of possibilities. Therefore, it is vital to achieve reliable and informative simulations that could potentially reduce experimental focus to a more manageable subset of candidates. That being said, it remains a challenge to predict properties, such as solid-state ionization energies, electron affinities, and charge carrier mobilities.

The morphology of the system is crucial in this task, as morphological disorder can lead to energetic disorder and energy traps, affecting charge transport. As a result, predictive structure-property simulations are difficult, as realistic morphologies require accurate 
modeling capable of predicting local packing arrangements, molecular ordering, and trap concentration. ${ }^{98}$ In order to construct morphologies representative of experimental systems, simulations require well controlled generation of homogenous amorphous solids. This can be achieved by thermal annealing above the glass transition temperature of the material, followed by a fast cooling process, to form a glass structure, in an attempt to imitate the typical deposition process of an OLED layer structure. By doing so, the molecules are locked in local energy minima. Unfortunately, this often varies significantly between experiment and simulation, such that the resulting morphologies regularly disagree. The main challenge is that realistic molecular packing is difficult to achieve and requires long simulation times to study self-assembly. By employing more complex methodology with increased computational cost, ultra-stable glass structures can be achieved by depositing particles one-by-one to obtain uniform packing, more closely mimicking that of experimental physical vapor deposition structures. ${ }^{99}$

Accurate morphologies rely on efficient classical force-fields, while accurate descriptions of morphological and electronic degrees of freedom require polarizable force-fields. Parametrization of such force fields is a tedious task and would be impossible for the vast number of organic compounds required. To overcome this, it is possible to construct building blocks made up of the essential components of compounds most likely to be experimentally investigated, thereby introducing the concept of an OLED material library.

\section{OLED simulation workflow}

First, in order to generate realistic morphologies, appropriate force fields including accurate descriptions of bonded and nonbonded interactions must be used. Additionally, polarizable force fields are required, which take into account the charge distribution rearrangement caused by changes in the environmental charge distribution. An amorphous morphology can then be simulated with molecular dynamics (MD), typically annealing above the glass transition temperature, followed by fast quenching to room temperature using the NPT ensemble. Density functional theory (DFT) based electronic structure methods can be utilized to compute gas-phase ionization energy $\left(\mathrm{IE}_{0}\right)$ and electron affinity $\left(\mathrm{EA}_{0}\right)$. The choice of functional has to be carefully considered with the importance of considering long-range corrected hybrid functionals for $\mathrm{IE}_{0}$ and $\mathrm{EA}_{0}$ values recently shown. ${ }^{100}$ In disordered organic materials, such as those found in OLEDs, charge carriers are localized and propagate through the system by successive hops from one molecule to another. Rates can be computed with the Miller-Abraham expression, ${ }^{101}$ typically utilized within Gaussian disorder models (GDM), with a lattice arrangement of hopping sites and Gaussian distributed site energies. Alternatively, rates can be described by a thermally activated type of transport in terms of the Marcus theory ${ }^{102-104}$ or by using Weiss-Dorsey rates ${ }^{105-109}$ for a wider range of temperature regimes, specifically using the low temperature approximations where Marcus rates are not applicable.

Within the high temperature limit of the classical charge transfer theory, ${ }^{103,110}$ the Marcus rate equation is derived from the importance of environmental coupling, using linear response theory to describe a heat bath coupled to electronic tunneling. ${ }^{105}$ This quantum mechanical tunneling moves the electron from one molecule to the other, at sufficiently high temperatures, when the nuclear vibrations (also described as bath fluctuations) bring the corresponding energy levels into resonance. The rate for a charge to hop from site $\mathrm{i}$ to site $\mathrm{j}\left(\omega_{\mathrm{ij}}\right)$ is given by

$$
\omega_{\mathrm{ij}}=\frac{2 \pi}{\hbar} \frac{\mathrm{J}_{\mathrm{ij}}^{2}}{\sqrt{4 \pi \lambda_{\mathrm{ij}} \mathrm{k}_{\mathrm{B}} \mathrm{T}}} \exp \left[-\frac{\left(\Delta \mathrm{E}_{\mathrm{ij}}-\lambda_{\mathrm{ij}}\right)^{2}}{4 \lambda_{\mathrm{ij}} \mathrm{k}_{\mathrm{B}} \mathrm{T}}\right],
$$

Here, $\lambda_{\mathrm{ij}}$ is the reorganization energy, the response to a change of charge state. $\mathrm{J}_{\mathrm{ij}}$ is the electronic coupling matrix elements, describing the strength of coupling between two localized states. $\Delta \mathrm{E}_{\mathrm{ij}}=\mathrm{E}_{\mathrm{i}}-\mathrm{E}_{\mathrm{j}}$ is the driving force, or site energy difference between two neighboring sites, where $E_{i}$ is the site energy of molecule i. ${ }^{111}$ Molecules within a small cutoff range are considered neighbors, between which carrier hopping can occur. The quantities within the Marcus equation are then computed for each neighboring pair. A brief outline is included below, and further details and an in-depth explanation of these calculations can be found elsewhere. ${ }^{54,112}$ Computation of site energies includes (1) the ionization energy and electron affinity of a single molecule in charged and neutral geometries and (2) the interaction with the environment, including the electrostatic and induction contributions, with adequate cutoffs for long-range interactions to be considered. For reorganization energies, considering a charging and discharging molecule, the internal contribution due to geometric rearrangement and external contribution due to the environment must both be included. Finally, the electronic coupling elements require approximation of diabatic states, usually with the ionization energy and electron affinity, for hole or electron transport, respectively. With the charge transfer rates computed, it is possible to then model charge dynamics. Each carrier hop or event takes the system from state "a" to " $b$ " with the corresponding rate for this transition and a probability for it to occur, which can be represented by the master equation. KMC is one method of solving the master equation that effectively provides charge carrier mobilities in the given system for holes or electrons. The complete multiscale simulation workflow is outlined in Fig. 5.

In order to facilitate a simpler workflow, it would be advantageous to have a library containing the vital components of these calculations. This would include the classical and polarizable force fields, as well as the structures and input parameters required for computation of electronic properties. In turn, this would establish the building blocks for further structures and systems to be investigated. In our current work, by creating these building blocks for various small molecule systems, it is our aim to provide an outlook for a fully functional OLED material library, which is of the upmost importance for future material design. In order for this concept to reach its full potential, i.e., the extraction of material properties from molecular structure alone, certain aspects of the forward problem should be addressed, which brings us to the adaptation of the material design strategy for future progress.

\section{THE FUTURE OF COMPUTATIONAL OLED DESIGN}

Ideally, parameter-free computer-based OLED design would be employed, utilizing pre-existing building blocks and tools, beginning with accurate prediction of material morphology for a new 


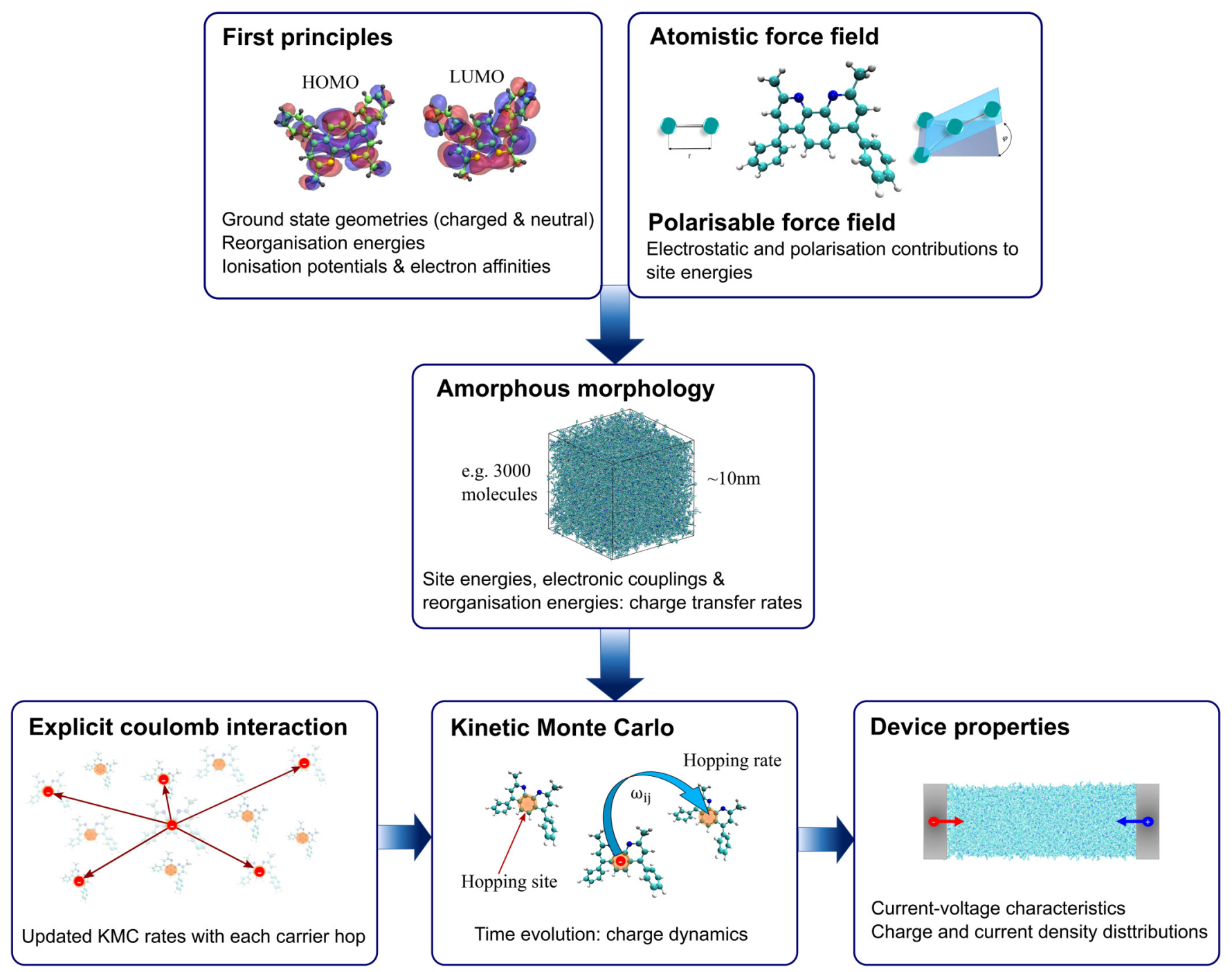

FIG. 5. OLED multiscale modeling simulation workflow: starting from first principles of an isolated molecule combined with atomistic and polarizable force fields. The amorphous morphology is generated with the use of molecular dynamics (MD). After computing the charge transfer rates, kinetic Monte Carlo (KMC) can then be used to solve the master equation to study charge dynamics (e.g., carrier mobilities), giving macroscopic device characteristics. Additionally, future development can allow for explicit coulomb interaction to be included within KMC; implementation would involve rate updates at each KMC step.

system followed by the calculation of the energetic landscape. This would lead to rate evaluation of the various processes within the system, such as charge or exciton transfer, proceeding to solving the time-dependent master equation, all of which provide essential quantities for material evaluation, such as electron affinity, ionization potential, and hole and electron mobilities. However, this multiscale procedure, with length and time scales spanning several orders of magnitude is non-trivial, and as such, it is difficult to predict device properties from the structure alone.

A necessity for future OLED development (and for all organic electronics) is the complete understanding of all fundamental processes within the device and constituent materials. Charge and energy transfer simulations play a pivotal role in this pursuit, thereby promoting the advancement of simulation techniques and methods to achieve an increasingly comprehensive description. Exciton formation and transfer are particularly important for OLED functionality. The theoretical tools used to determine exciton transport parameters include DFT for electronic excitation properties, ${ }^{113}$ many-body Green's function theory, and GW approximation with the BetheSalpeter equation $(\mathrm{GW}-\mathrm{BSE})^{114}$ for excited states ${ }^{113,115,116}$ and an adaption of Marcus theory to describe exciton diffusion. ${ }^{117}$ Further to this, an essential link between micro and macroscale would be 
employing more realistic charge transport dynamics, with an important addition, but computationally demanding approach, being the inclusion of explicit coulomb interactions and reevaluation of charge transfer rates at each KMC step.

The embedding of electrostatics within KMC simulations, as shown in Fig. 5, would provide valuable insight into charge dynamics, specifically, within slab geometries and interface interactions, vital for device characteristics. However, when investigating a system that is stochastically propagated in time, with each charge carrier hop leading to a new charge distribution and consequently altering all interactions with other charge carriers, the complexity of the problem is elevated and requires accurate and efficient methodology.

The accurate evaluation of electrostatic interactions is crucial but still remains a challenge as the methods to compute electrostatics require large system sizes and inherently long-range (complex) interactions, leading to high computational cost. Interaction distance cutoff methods can be applied to reduce the electrostatic contributions. But due to the long-range nature of electrostatics, as a result of the $1 / \mathrm{r}$ decay of the Coulomb potential, a cutoff radius is often insufficient and more exact methods have to be employed. In fact, neglecting long-range contributions has been shown to cause inaccuracies with variation of simulated carrier densities and device performance predictions, when compared to exact methods. ${ }^{118}$ On the other hand, using exact methods for computing electrostatics typically involves summation techniques, such as Ewald summation $^{119}$ and the more efficient particle mesh Ewald (PME). ${ }^{120,121}$ However, in large periodic systems, an exact description is often unfeasible or highly computationally demanding. Therefore, further methods have to be considered, specifically for nonperiodic systems (requiring more sophisticated summation).

Very recently, efficient electrostatic evaluation for the use in KMC applications have been demonstrated by exploiting the fact that each KMC step corresponds to one charge carrier hop, resulting in a modest charge redistribution. ${ }^{122,123}$ This has been achieved with a new variant of the Fast Multipole Method ${ }^{122}$ and in a second approach utilizing local charge contributions to the hopping rate before and after a hop, allowing for a newly adapted cutoff scheme. ${ }^{123}$ The implementation of electrostatics within KMC simulations is clearly challenging, but this recent progress shows it is achievable.

Furthermore, computational material design tools look toward other future developments. A key area and one which has gained significant interest in recent years is machine learning based techniques. Application of machine learning for OLED materials would be a significant step forward for their computational design. For this to be achievable, accurate molecular/chemical descriptors are crucial. This is a difficult task, as it requires identification of correlations between similar structures with similar properties, linking this to a simple and systematic feature which can be extracted. ${ }^{124}$ For OLED materials, these vital descriptors are missing, and only when they can be accurately obtained can the field move toward machine learning approaches. Nevertheless, recent progress in machine learning demonstrates the significant potential of these methods. First, local properties such as electrostatic multipoles can already be predicted using kernel-based techniques. ${ }^{125}$ Secondly, they can present practical strategies to parametrize force fields by providing coarse-grained (CG) potentials, which are more efficient. ${ }^{126}$ Third, properties such as the glass transition temperature can be correlated with the chemical structure, using a quantitative structure-property relationship approach, ${ }^{127}$ with predictive modeling capable of pre-screening thermally stable candidates from only topological indicies, ${ }^{128}$ altogether highlighting the possibilities of machine learning in the context of OLED design.

\section{CONCLUSION AND OUTLOOK}

In summary, OLED applications do offer a unique alternative to traditional display technologies, with their flexible, lightweight, and transparent possibilities. However, due to their commercial infancy, they have obstacles to overcome, particularly regarding device lifetime and efficiency. Within this Perspective, we have highlighted the improvements made to each of the OLED layers, with respect to their constituent materials, most notably the emissive layer and the extensive efforts to achieve an efficient and stable blue OLED, including the novel UPSF approach, bringing a blue OLED to light by reaching a balance of stability, efficiency, and emissive color, accompanied by our multiscale computational studies, highlighting the possibilities of the concept with an appropriate material design. The shift to an OLED material library then signifies the importance to achieve pre-screening, reducing an almost infinite set of potential candidates for OLED applications, to a more manageable number prior to synthesis. This multiscale procedure is, of course, a non-trivial task, and certain aspects of the forward problem need to be addressed before moving to parameter-free methods, allowing for extraction of device properties from the molecular structure alone. Computational method development is therefore essential, only then can the gap between structure and device properties be closed, thereby reducing both experimental and computational cost.

\section{ACKNOWLEDGMENTS}

D.A. acknowledges the BMBF Grant InterPhase (No. FKZ 13N13661) and the European Union Horizon 2020 Research and Innovation Programme 'Widening Materials Models' under Grant Agreement No. 646259 (MOSTOPHOS). This research has been supported by the King Abdullah University of Science and Technology (KAUST), via the Competitive Research Grants (CRG) Program. D.A. acknowledges KAUST for hosting his sabbatical.

\section{DATA AVAILABILITY}

Data sharing is not applicable to this article as no new data were created or analyzed in this study.

\section{REFERENCES}

${ }^{1}$ A. Bernanose, M. Comte, and P. Vouaux, "A new method of emission of light by certain organic compounds," J. Chim. Phys. 50, 64-68 (1953).

$\mathbf{2}$ A. Bernanose and P. Vouaux, "Electroluminescence of organic compounds," J. Chim. Phys. 50, 261-263 (1953).

${ }^{3}$ M. Pope, H. P. Kallmann, and P. Magnante, "Electroluminescence in organic crystals,” J. Chem. Phys. 38(8), 2042-2043 (1963).

${ }^{4}$ C. W. Tang and S. A. VanSlyke, "Organic electroluminescent diodes," Appl. Phys. Lett. 51, 913 (1987). 
${ }^{5}$ P. Heimel, A. Mondal, F. May, W. Kowalsky, C. Lennartz, D. Andrienko, and R. Lovrincic, "Unicolored phosphor-sensitized fluorescence for efficient and stable blue OLEDs," Nat. Commun. 9(1), 4990 (2018).

${ }^{6}$ C. Eickhoff, P. Murer, T. Geßner, J. Birnstock, M. Kröger, Z. Choi, S. Watanabe, F. May, C. Lennartz, I. Stengel, I. Münster, K. Kahle, G. Wagenblast, and H. Mangold, "Long-lived and highly efficient green and blue phosphorescent emitters and device architectures for OLED displays," in Organic Light Emitting Materials and Devices XIX (International Society for Optics and Photonics, 2015), Vol. 9566, p. 95662N.

${ }^{7}$ C. W. Tang, S. A. VanSlyke, and C. H. Chen, "Electroluminescence of doped organic thin films," J. Appl. Phys. 65(9), 3610-3616 (1989).

${ }^{8}$ A. P. Kulkarni, C. J. Tonzola, A. Babel, and S. A. Jenekhe, "Electron transport materials for organic light-emitting diodes," Chem. Mater. 16(23), 4556-4573 (2004).

${ }^{99}$ A. Udhiarto, Y. Sister, S. Rini, M. Asvial, and B. Munir, "Effect of hole transport layer and electron transport layer on the performance of a single emissive layer organic light emitting diode," in International Conference on Quality in Research (QiR) (IEEE, Lombok, 2015), pp. 137-140. .

${ }^{10} \mathrm{C}$. Féry, B. Racine, D. Vaufrey, H. Doyeux, and S. Cinà, "Physical mechanism responsible for the stretched exponential decay behavior of aging organic lightemitting diodes," Appl. Phys. Lett. 87(21), 213502 (2005).

${ }^{11}$ T. D. Schmidt, L. Jäger, Y. Noguchi, H. Ishii, and W. Brütting, "Analyzing degradation effects of organic light-emitting diodes via transient optical and electrical measurements," J. Appl. Phys. 117(21), 215502 (2015).

${ }^{12}$ D. Y. Kondakov and R. H. Young, "Variable sensitivity of organic lightemitting diodes to operation-induced chemical degradation: Nature of the antagonistic relationship between lifetime and efficiency," J. Appl. Phys. 108(7), 074513 (2010).

${ }^{13}$ S. Scholz, D. Kondakov, B. Lüssem, and K. Leo, "Degradation mechanisms and reactions in organic light-emitting devices," Chem. Rev. 115(16), 8449-8503 (2015).

${ }^{14}$ C. Adachi, M. A. Baldo, S. R. Forrest, S. Lamansky, M. E. Thompson, and R. C. Kwong, "High-efficiency red electrophosphorescence devices," Appl. Phys. Lett. 78(11), 1622-1624 (2001).

${ }^{15}$ D. F. O'Brien, M. A. Baldo, M. E. Thompson, and S. R. Forrest, "Improved energy transfer in electrophosphorescent devices," Appl. Phys. Lett. 74(3), 442-444 (1999).

${ }^{16}$ M. A. Baldo, D. F. O’Brien, Y. You, A. Shoustikov, S. Sibley, M. E. Thompson, and S. R. Forrest, "Highly efficient phosphorescent emission from organic electroluminescent devices," Nature 395, 151 (1998).

${ }^{17}$ Y.-L. Tung, P.-C. Wu, C.-S. Liu, Y. Chi, J.-K. Yu, Y.-H. Hu, P.-T. Chou, S.-M. Peng, G.-H. Lee, Y. Tao, A. J. Carty, C.-F. Shu, and F.-I. Wu, "Highly efficient red phosphorescent osmium(II) complexes for OLED applications," Organometallics 23(15), 3745-3748 (2004).

${ }^{18} \mathrm{H}$. Fujii, H. Sakurai, K. Tani, K. Wakisaka, and T. Hirao, "Bright and ultimately pure red electrophosphorescent diode bearing diphenylquinoxaline," IEICE Electron. Express 2(8), 260-266 (2005).

${ }^{19} \mathrm{H}$. Kanno, K. Ishikawa, Y. Nishio, A. Endo, C. Adachi, and K. Shibata, "Highly efficient and stable red phosphorescent organic light-emitting device using bis[2-(2-benzothiazoyl)phenolato]zinc(II) as host material," Appl. Phys. Lett. 90(12), 123509 (2007)

${ }^{\mathbf{2 0}} \mathrm{R}$. Meerheim, K. Walzer, M. Pfeiffer, and K. Leo, "Ultrastable and efficient red organic light emitting diodes with doped transport layers," Appl. Phys. Lett. 89(6), 061111 (2006).

${ }^{21}$ R. Furue, K. Matsuo, Y. Ashikari, H. Ooka, N. Amanokura, and T. Yasuda, "Highly efficient red-orange delayed fluorescence emitters based on strong $\pi$-accepting dibenzophenazine and dibenzoquinoxaline cores: Toward a rational pure-red OLED design," Adv. Opt. Mater. 6(5), 1701147 (2018).

${ }^{22}$ F. Ni, Z. Wu, Z. Zhu, T. Chen, K. Wu, C. Zhong, K. An, D. Wei, D. Ma, and C. Yang, "Teaching an old acceptor new tricks: Rationally employing 2,1,3-benzothiadiazole as input to design a highly efficient red thermally activated delayed fluorescence emitter," J. Mater. Chem. C 5(6), 1363-1368 (2017).
${ }^{23}$ S. Wang, X. Yan, Z. Cheng, H. Zhang, Y. Liu, and Y. Wang, "Highly efficient near-infrared delayed fluorescence organic light emitting diodes using a phenanthrene-based charge-transfer compound," Angew. Chem. Int. Ed. 54(44), 13068-13072 (2015).

${ }^{24}$ M. A. Baldo, S. Lamansky, P. E. Burrows, M. E. Thompson, and S. R. Forrest, "Very high-efficiency green organic light-emitting devices based on electrophosphorescence," Appl. Phys. Lett. 75(1), 4-6 (1999).

${ }^{25}$ H.-Y. Li, L. Zhou, M.-Y. Teng, Q.-L. Xu, C. Lin, Y.-X. Zheng, J.-L. Zuo, H.-J. Zhang, and X.-Z. You, "Highly efficient green phosphorescent OLEDs based on a novel iridium complex," J. Mater. Chem. C 1(3), 560-565 (2013).

${ }^{\mathbf{2 6}}$ Y. Tao, Q. Wang, C. Yang, C. Zhong, J. Qin, and D. Ma, "Multifunctional triphenylamine/oxadiazole hybrid as host and exciton-blocking material: High efficiency green phosphorescent OLEDs using easily available and common materials," Adv. Funct. Mater. 20(17), 2923-2929 (2010).

${ }^{27}$ C.-H. Chang, M.-C. Kuo, W.-C. Lin, Y.-T. Chen, K.-T. Wong, S.-H. Chou, E. Mondal, R. C. Kwong, S. Xia, T. Nakagawa, and C. Adachi, "A dicarbazoletriazine hybrid bipolar host material for highly efficient green phosphorescent OLEDs," J. Mater. Chem. 22(9), 3832 (2012).

${ }^{28}$ H.-F. Chen, S.-J. Yang, Z.-H. Tsai, W.-Y. Hung, T.-C. Wang, and K.-T. Wong, "1,3,5-Triazine derivatives as new electron transport-type host materials for highly efficient green phosphorescent OLEDs," J. Mater. Chem. 19(43), 8112 (2009).

${ }^{29}$ C. Wang, Y.-Q. Zheng, J. Tang, J.-L. Yu, F. Yang, B. Wei, X.-F. Li, and J.-H. Zhang, "Highly efficient green TADF organic light-emitting diodes by simultaneously manipulating hole and electron transport," Nanotechnology 30(11), 115201 (2019).

${ }^{30}$ H. Tanaka, K. Shizu, H. Miyazaki, and C. Adachi, "Efficient green thermally activated delayed fluorescence (TADF) from a phenoxazine-triphenyltriazine (PXZ-TRZ) derivative," Chem. Commun. 48(93), 11392 (2012).

${ }^{31}$ B. S. Kim and J. Y. Lee, "Engineering of mixed host for high external quantum efficiency above $25 \%$ in green thermally activated delayed fluorescence device," Adv. Funct. Mater. 24(25), 3970-3977 (2014).

${ }^{32} \mathrm{Y}$. Im and J. Y. Lee, "Recent progress of green thermally activated delayed fluorescent emitters," J. Inf. Disp. 18(3), 101-117 (2017).

${ }^{33}$ Y. Seino, S. Inomata, H. Sasabe, Y.-J. Pu, and J. Kido, "High-performance green OLEDs using thermally activated delayed fluorescence with a power efficiency of over $100 \mathrm{Lm} \mathrm{W}^{-1}$, Adv. Mater. 28(13), 2638-2643 (2016).

${ }^{34}$ J.-H. Lee, C.-H. Chen, P.-H. Lee, H.-Y. Lin, M. Leung, T.-L. Chiu, and C.-F. Lin, "Blue organic light-emitting diodes: Current status, challenges, and future outlook,” J. Mater. Chem. C 7(20), 5874-5888 (2019).

${ }^{35} \mathrm{H}$. Kuma and C. Hosokawa, "Blue fluorescent OLED materials and their application for high-performance devices," Sci. Technol. Adv. Mater. 15(3), 034201 (2014).

${ }^{36}$ J. Lee, H.-F. Chen, T. Batagoda, C. Coburn, P. I. Djurovich, M. E. Thompson, and S. R. Forrest, "Deep blue phosphorescent organic light-emitting diodes with very high brightness and efficiency," Nat. Mater. 15(1), 92-98 (2016).

${ }^{37} \mathrm{~K}$. Klimes, Z. Zhu, and J. Li, "Efficient blue phosphorescent OLEDs with improved stability and color purity through judicious triplet exciton management," Adv. Funct. Mater. 29(31), 1903068 (2019).

${ }^{38} \mathrm{~S}$. K. Jeon and J. Y. Lee, "Four times lifetime improvement of blue phosphorescent organic light-emitting diodes by managing recombination zone," Org. Electron. 27, 202-206 (2015).

${ }^{39}$ S. Hirata, Y. Sakai, K. Masui, H. Tanaka, S. Y. Lee, H. Nomura, N. Nakamura M. Yasumatsu, H. Nakanotani, Q. Zhang, K. Shizu, H. Miyazaki, and C. Adachi, "Highly efficient blue electroluminescence based on thermally activated delayed fluorescence," Nat. Mater. 14(3), 330-336 (2015).

${ }^{40} \mathrm{H}$. Uoyama, K. Goushi, K. Shizu, H. Nomura, and C. Adachi, "Highly efficient organic light-emitting diodes from delayed fluorescence," Nature 492(7428), 234-238 (2012).

${ }^{41}$ T.-T. Bui, F. Goubard, M. Ibrahim-Ouali, D. Gigmes, and F. Dumur, "Recent advances on organic blue thermally activated delayed fluorescence (TADF) emitters for organic light-emitting diodes (OLEDs)," Beilstein J. Org. Chem. 14, 282-308 (2018). 
${ }^{42}$ P. L. dos Santos, J. S. Ward, M. R. Bryce, and A. P. Monkman, "Using guesthost interactions to optimize the efficiency of TADF OLEDs," J. Phys. Chem. Lett. 7(17), 3341-3346 (2016).

${ }^{43}$ V. Jankus, P. Data, D. Graves, C. McGuinness, J. Santos, M. R. Bryce, F. B. Dias, and A. P. Monkman, "Highly efficient TADF OLEDs: How the emitter-host interaction controls both the excited state species and electrical properties of the devices to achieve near $100 \%$ triplet harvesting and high efficiency," Adv. Funct. Mater. 24(39), 6178-6186 (2014).

${ }^{44}$ P. Rajamalli, N. Senthilkumar, P. Gandeepan, C.-C. Ren-Wu, H.-W. Lin, and C.-H. Cheng, "A method for reducing the singlet-triplet energy gaps of TADF materials for improving the blue OLED efficiency," ACS Appl. Mater. Interfaces 8(40), 27026-27034 (2016).

${ }^{45}$ R. Seifert, I. Rabelo de Moraes, S. Scholz, M. C. Gather, B. Lüssem, and K. Leo, "Chemical degradation mechanisms of highly efficient blue phosphorescent emitters used for organic light emitting diodes," Org. Electron. 14(1), 115-123 (2013).

${ }^{46}$ S. Kim, H. J. Bae, S. Park, W. Kim, J. Kim, J. S. Kim, Y. Jung, S. Sul, S.-G. Ihn, C. Noh, S. Kim, and Y. You, "Degradation of blue-phosphorescent organic lightemitting devices involves exciton-induced generation of polaron pair within emitting layers," Nat. Commun. 9(1), 1211 (2018).

${ }^{47} \mathrm{Y}$. Zhang and H. Aziz, "Degradation mechanisms in blue phosphorescent organic light-emitting devices by exciton-polaron interactions: Loss in quantum yield versus loss in charge balance," ACS Appl. Mater. Interfaces 9(1), 636-643 (2017).

${ }^{48} \mathrm{~W}$. Song and J. Y. Lee, "Degradation mechanism and lifetime improvement strategy for blue phosphorescent organic light-emitting diodes," Adv. Opt. Mater. 5(9), 1600901 (2017).

${ }^{49}$ M. A. Baldo, M. E. Thompson, and S. R. Forrest, "High-efficiency fluorescent organic light-emitting devices using a phosphorescent sensitizer," Nature 403(6771), 750-753 (2000).

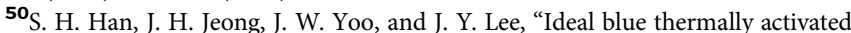
delayed fluorescence emission assisted by a thermally activated delayed fluorescence assistant dopant through a fast reverse intersystem crossing mediated cascade energy transfer process," J. Mater. Chem. C 7(10), 3082-3089 (2019).

${ }^{51}$ R. Coehoorn, H. van Eersel, P. Bobbert, and R. Janssen, "Kinetic Monte Carlo study of the sensitivity of OLED efficiency and lifetime to materials parameters," Adv. Funct. Mater. 25(13), 2024-2037 (2015).

${ }^{52}$ J. Cottaar, R. Coehoorn, and P. A. Bobbert, "Modeling of charge transport across disordered organic heterojunctions," Org. Electron. 13(4), 667-672 (2012).

${ }^{53}$ P. Friederich, A. Fediai, S. Kaiser, M. Konrad, N. Jung, and W. Wenzel, "Toward design of novel materials for organic electronics," Adv. Mater. 31(26), 1808256 (2019).

${ }^{54}$ P. Kordt, J. J. M. van der Holst, M. Al Helwi, W. Kowalsky, F. May, A. Badinski, C. Lennartz, and D. Andrienko, "Modeling of organic light emitting diodes: From molecular to device properties," Adv. Funct. Mater. 25(13), 1955-1971 (2015)

${ }^{55}$ B. Baumeier, F. May, C. Lennartz, and D. Andrienko, "Challenges for in silico design of organic semiconductors,” J. Mater. Chem. 22(22), 10971 (2012).

${ }^{56}$ L. Duan, K. Xie, and Y. Qiu, "Review paper: Progress on efficient cathodes for organic light-emitting diodes,” J. Soc. Inf. Disp. 19(6), 453 (2011).

${ }^{57}$ Y. Kawamura, S. Yanagida, and S. R. Forrest, "Energy transfer in polymer electrophosphorescent light emitting devices with single and multiple doped luminescent layers," J. Appl. Phys. 92(1), 87-93 (2002).

${ }^{58}$ X. Zhou, D. S. Qin, M. Pfeiffer, J. Blochwitz-Nimoth, A. Werner, J. Drechsel, B. Maennig, K. Leo, M. Bold, P. Erk, and H. Hartmann, "High-efficiency electrophosphorescent organic light-emitting diodes with double light-emitting layers," Appl. Phys. Lett. 81(21), 4070-4072 (2002).

${ }^{59}$ L. S. Hung, C. W. Tang, and M. G. Mason, "Enhanced electron injection in organic electroluminescence devices using an $\mathrm{Al} / \mathrm{LiF}$ electrode," Appl. Phys. Lett. 70(2), 152-154 (1997).

${ }^{60} \mathrm{H}$. J. Bolink, E. Coronado, D. Repetto, and M. Sessolo, "Air stable hybrid organic-inorganic light emitting diodes using $\mathrm{ZnO}$ as the cathode," Appl. Phys. Lett. 91(22), 223501 (2007).
${ }^{61}$ Y.-J. Pu, M. Miyamoto, K-I. Nakayama, T. Oyama, Y. Masaaki, and J. Kido, "Lithium phenolate complexes for an electron injection layer in organic lightemitting diodes," Org. Electron. 10(2), 228-232 (2009).

${ }^{62}$ P.-C. Kao, J.-H. Lin, J.-Y. Wang, C.-H. Yang, and S.-H. Chen, "Improved electron injection into Alq3 based OLEDs using a thin lithium carbonate buffer layer," Synth. Met. 160(15-16), 1749-1753 (2010).

${ }^{63} \mathrm{H}$. Sasabe and J. Kido, "Low molecular weight materials electron-transport materials," in Handbook of Organic Light-Emitting Diodes, edited by C. Adachi, R. Hattori, H. Kaji, and T. Tsujimura (Springer Japan, Tokyo, 2019), pp. 1-10.

${ }^{64}$ N. B. Kotadiya, A. Mondal, P. W. M. Blom, D. Andrienko, and G.-J. A. $\mathrm{H}$. Wetzelaer, "A window to trap-free charge transport in organic semiconducting thin films," Nat. Mater. 18(11), 1182-1186 (2019).

${ }^{65} \mathrm{~V}$. A. Montes, C. Pérez-Bolívar, L. A. Estrada, J. Shinar, and P. Anzenbacher, "Ultrafast dynamics of triplet excitons in $\mathrm{Alq}_{3}$-bridge- $\mathrm{Pt}(\mathrm{II})$ porphyrin electroluminescent materials," J. Am. Chem. Soc. 129(42), 12598-12599 (2007).

${ }^{66}$ C.-M. Keum, N. M. Kronenberg, C. Murawski, K. Yoshida, Y. Deng, C. Berz, W. Li, M. Wei, I. D. W. Samuel, and M. C. Gather, "The role of metallic dopants in improving the thermal stability of the electron transport layer in organic lightemitting diodes," Adv. Opt. Mater. 6(17), 1800496 (2018).

${ }^{67}$ N. B. Kotadiya, H. Lu, A. Mondal, Y. Ie, D. Andrienko, P. W. M. Blom, and G.-J. A. H. Wetzelaer, "Universal strategy for ohmic hole injection into organic semiconductors with high ionization energies," Nat. Mater. 17(4), 329-334 (2018).

${ }^{68}$ Y. Shirota, Y. Kuwabaraa, D. Okuda, R. Okuda, H. Ogawa, H. Inada, T. Wakimoto, H. Nakada, Y. Yonemoto, S. Kawami, and K. Imai, "Starburst molecules based on $\pi$-electron systems as materials for organic electroluminescent devices," J. Lumin. 72-74, 985-991 (1997).

${ }^{69}$ S. Shahnawaz, S. Sudheendran Swayamprabha, M. R. Nagar, R. A. K. Yadav, S. Gull, D. K. Dubey, and J.-H. Jou, "Hole-transporting materials for organic light-emitting diodes: An overview," J. Mater. Chem. C 7(24), 7144-7158 (2019).

${ }^{70}$ J. S. Martins, A. A. Bartolomeu, W. H. dos Santos, L. C. da Silva Filho, E. F. de Oliveira, F. C. Lavarda, A. Cuin, C. Legnani, I. O. Maciel, B. Fragneaud, and W. G. Quirino, "New class of organic hole-transporting materials based on xanthene derivatives for organic electronic applications," J. Phys. Chem. C 121(24), 12999-13007 (2017)

${ }^{71}$ Z. Zheng, Q. Dong, L. Gou, J.-H. Su, and J. Huang, "Novel hole transport

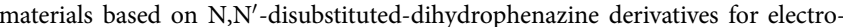
luminescent diodes," J. Mater. Chem. C 2(46), 9858-9865 (2014).

${ }^{72}$ Q. Zhang, J. Chen, Y. Cheng, L. Wang, D. Ma, X. Jing, and F. Wang, "Novel hole-transporting materials based on 1,4-bis(carbazolyl)benzene for organic light-emitting devices," J. Mater. Chem. 14(5), 895 (2004).

${ }^{73}$ T. D. Schmidt, T. Lampe, D. M. R. Sylvinson, P. I. Djurovich, M. E. Thompson, and W. Brütting, "Emitter orientation as a key parameter in organic light-emitting diodes,” Phys. Rev. Appl. 8(3), 037001 (2017).

${ }^{74}$ C.-K. Moon, K.-H. Kim, J. W. Lee, and J.-J. Kim, "Influence of host molecules on emitting dipole orientation of phosphorescent iridium complexes," Chem. Mater. 27(8), 2767-2769 (2015)

${ }^{75}$ W. Brütting, Physics of Organic Semiconductors (WILEY_VCH Verlag GmbH \& Co. KGaA, Weinheim, 2005)

${ }^{76}$ Y. Wang, W. Wang, Z. Huang, H. Wang, J. Zhao, J. Yu, and D. Ma, "High-efficiency red organic light-emitting diodes based on a double-emissive layer with an external quantum efficiency over 30\%," J. Mater. Chem. C 6(26), 7042-7045 (2018).

${ }^{77}$ Q. Yang, Y. Hao, Z. Wang, Y. Li, H. Wang, and B. Xu, "Double-emission-layer green phosphorescent OLED based on LiF-doped TPBi as electron transport layer for improving efficiency and operational lifetime," Synth. Met. 162(3-4), 398-401 (2012).

${ }^{78}$ H. van Eersel, P. A. Bobbert, R. A. J. Janssen, and R. Coehoorn, "Monte Carlo study of efficiency roll-off of phosphorescent organic light-emitting diodes: Evidence for dominant role of triplet-polaron quenching," Appl. Phys. Lett. 105(14), 143303 (2014)

${ }^{79}$ F. X. Zang, T. C. Sum, A. C. H. Huan, T. L. Li, W. L. Li, and F. Zhu, "Reduced efficiency roll-off in phosphorescent organic light emitting diodes at ultrahigh 
current densities by suppression of triplet-polaron quenching," Appl. Phys. Lett. 93(2), 023309 (2008).

${ }^{80} \mathrm{M}$. A. Baldo, C. Adachi, and S. R. Forrest, "Transient analysis of organic electrophosphorescence. II. Transient analysis of triplet-triplet annihilation," Phys. Rev. B 62(16), 10967-10977 (2000).

${ }^{81}$ S. Reineke, G. Schwartz, K. Walzer, and K. Leo, "Reduced efficiency roll-off in phosphorescent organic light emitting diodes by suppression of triplet-triplet annihilation," Appl. Phys. Lett. 91(12), 123508 (2007).

${ }^{82}$ Y. Luo and H. Aziz, "Probing triplet-triplet annihilation zone and determining triplet exciton diffusion length by using delayed electroluminescence," J. Appl. Phys. 107(9), 094510 (2010).

${ }^{83}$ F. May, M. Al-Helwi, B. Baumeier, W. Kowalsky, E. Fuchs, C. Lennartz, and D. Andrienko, "Design rules for charge-transport efficient host materials for phosphorescent organic light-emitting diodes," J. Am. Chem. Soc. 134(33), 13818-13822 (2012)

${ }^{84} \mathrm{M}$. Cai, D. Zhang, and L. Duan, "High performance thermally activated delayed fluorescence sensitized organic light-emitting diodes," Chem. Rec. 19(8), 1611-1623 (2019).

${ }^{85}$ H. Nakanotani, T. Higuchi, T. Furukawa, K. Masui, K. Morimoto, M. Numata, H. Tanaka, Y. Sagara, T. Yasuda, and C. Adachi, "High-efficiency organic light-emitting diodes with fluorescent emitters," Nat. Commun. 5(1), 4016 (2014).

${ }^{86} \mathrm{~K}$. H. Lee and J. Y. Lee, "Phosphor sensitized thermally activated delayed fluorescence organic light-emitting diodes with ideal deep blue device performances," J. Mater. Chem. C 7(28), 8562-8568 (2019).

${ }^{87}$ B. W. D'Andrade, M. A. Baldo, C. Adachi, J. Brooks, M. E. Thompson, and S. R. Forrest, "High-efficiency yellow double-doped organic light-emitting devices based on phosphor-sensitized fluorescence," Appl. Phys. Lett. 79(7), 1045-1047 (2001).

${ }^{88}$ G. Lei, L. Wang, and Y. Qiu, "Blue phosphorescent dye as sensitizer and emitter for white organic light-emitting diodes," Appl. Phys. Lett. 85(22), 5403-5405 (2004)

${ }^{89}$ G. Cheng, Y. Zhang, Y. Zhao, S. Liu, and Y. Ma, "Improved efficiency for white organic light-emitting devices based on phosphor sensitized fluorescence," Appl. Phys. Lett. 88(8), 083512 (2006).

${ }^{90} \mathrm{H}$. Kanno, Y. Sun, and S. R. Forrest, "White organic light-emitting device based on a compound fluorescent-phosphor-sensitized-fluorescent emission layer," Appl. Phys. Lett. 89(14), 143516 (2006).

${ }^{91}$ L. Paterson, A. Mondal, P. Heimel, R. Lovrincic, F. May, C. Lennartz, and D. Andrienko, "Perspectives of unicolored phosphor-sensitized fluorescence," Adv. Electron. Mater. 5(12), 1900646 (2019).

${ }^{92}$ S. Schmidbauer, A. Hohenleutner, and B. König, "Chemical degradation in organic light-emitting devices: Mechanisms and implications for the design of new materials," Adv. Mater. 25(15), 2114-2129 (2013).

${ }^{93} \mathrm{Q}$. Wang and H. Aziz, "Exciton-polaron-induced aggregation of organic electroluminescent materials: A major degradation mechanism in wide-bandgap phosphorescent and fluorescent organic light-emitting devices," Adv. Opt. Mater. 3(7), 967-975 (2015).

${ }^{94}$ M. Hong, M. K. Ravva, P. Winget, and J.-L. Brédas, "Effect of substituents on the electronic structure and degradation process in carbazole derivatives for blue OLED host materials," Chem. Mater. 28(16), 5791-5798 (2016).

${ }^{95}$ J. Gibson, A. P. Monkman, and T. J. Penfold, "The importance of vibronic coupling for efficient reverse intersystem crossing in thermally activated delayed fluorescence molecules," ChemPhysChem 17(19), 2956-2961 (2016).

${ }^{96} \mathrm{X}$. de Vries, R. Coehoorn, and P. A. Bobbert, "High energy acceptor states strongly enhance exciton transfer between metal organic phosphorescent dyes," Nat. Commun. 11(1), 1292 (2020).

${ }^{97}$ S. Zeder, C. Kirsch, U. Aeberhard, B. Blülle, S. Jenatsch, and B. Ruhstaller, "Coupled 3D master equation and 1D drift-diffusion approach for advanced OLED modeling," J. Soc. Inf. Disp. 28(5), 440-449 (2020).

${ }^{98} \mathrm{D}$. Andrienko, "Simulations of morphology and charge transport in supramolecular organic materials," in Smart Materials Series, edited by N. Koch (Royal Society of Chemistry, Cambridge, 2014), Chap. 9, pp. 309-362.
${ }^{99}$ S. Singh, M. D. Ediger, and J. J. de Pablo, "Ultrastable glasses from in silico vapour deposition," Nat. Mater. 12(2), 139-144 (2013).

${ }^{100}$ L. Gallandi and T. Körzdörfer, "Long-range corrected DFT meets GW: Vibrationally resolved photoelectron spectra from first principles," J. Chem. Theory Comput. 11(11), 5391-5400 (2015).

${ }^{101}$ A. Miller and E. Abrahams, "Impurity conduction at low concentrations," Phys. Rev. 120(3), 745-755 (1960).

${ }^{102}$ R. A. Marcus, "Chemical and electrochemical electron-transfer theory," Annu. Rev. Phys. Chem. 15(1), 155-196 (1964).

${ }^{103}$ R. A. Marcus, "Electron transfer reactions in chemistry: Theory and experiment (nobel lecture)," Angew. Chem. Int. Ed. Engl. 32(8), 1111-1121 (1993).

${ }^{104}$ R. A. Marcus and N. Sutin, "Electron transfers in chemistry and biology," Biophys. Acta 811(3), 265-322 (1985).

${ }^{105}$ R. Egger, C. H. Mak, and U. Weiss, "Quantum rates for nonadiabatic electron transfer,” J. Chem. Phys. 100(4), 2651-2660 (1994).

${ }^{106} \mathrm{H}$. Grabert and U. Weiss, "Thermal enhancement of the quantum decay rate in a dissipative system,” Z. Phys. B Condens. Matter 56(2), 171-183 (1984).

${ }^{107} \mathrm{H}$. Grabert and U. Weiss, "Quantum tunneling rates for asymmetric doublewell systems with ohmic dissipation," Phys. Rev. Lett. 54(15), 1605-1608 (1985).

${ }^{108}$ M. P. A. Fisher and A. T. Dorsey, "Dissipative quantum tunneling in a biased double-well system at finite temperatures," Phys. Rev. Lett. 54(15), 1609-1612 (1985).

${ }^{109}$ H. Grabert, P. Olschowski, and U. Weiss, "Quantum decay rates for dissipative systems at finite temperatures,” Phys. Rev. B 36(4), 1931-1951 (1987).

${ }^{110}$ G. R. Hutchison, M. A. Ratner, and T. J. Marks, "Hopping transport in conductive heterocyclic oligomers: Reorganization energies and substituent effects," Am. Chem. Soc. 127(7), 2339-2350 (2005).

${ }^{111}$ V. May and O. Kühn, Charge and Energy Transfer Dynamics in Molecular Systems (Wiley-VCH, Weinheim, 2011).

${ }^{112}$ V. Rühle, A. Lukyanov, F. May, M. Schrader, T. Vehoff, J. Kirkpatrick, B. Baumeier, and D. Andrienko, "Microscopic simulations of charge transport in disordered organic semiconductors," J. Chem. Theory Comput. 7(10), 3335-3345 (2011).

${ }^{113}$ C. Faber, P. Boulanger, C. Attaccalite, I. Duchemin, and X. Blase, "Excited states properties of organic molecules: From density functional theory to the GW and Bethe-Salpeter Green's function formalisms," Philos. Trans. R. Soc. A 372, 20130271 (2014).

${ }^{114} \mathrm{~L}$. Hedin and S. Lundqvist, "Effects of electron-electron and electron-phonon interactions on the one-electron states of solids," Solid State Phys. 23, 1-181 (1970).

${ }^{115}$ B. Bagheri, B. Baumeier, and M. Karttunen, "Getting excited: Challenges in quantum-classical studies of excitons in polymeric systems," Phys. Chem. Chem. Phys. 18(44), 30297-30304 (2016).

${ }^{116} \mathrm{Y}$. Jin and W. Yang, "Excitation energies from the single-particle Green's function with the $G W$ approximation," J. Phys. Chem. A 123(14), 3199-3204 (2019).

${ }^{117}$ V. Stehr, R. F. Fink, B. Engels, J. Pflaum, and C. Deibel, "Singlet exciton diffusion in organic crystals based on Marcus transfer rates," J. Chem. Theory Comput. 10(3), 1242-1255 (2014).

${ }^{118}$ M. Casalegno, G. Raos, and R. Po, "Methodological assessment of kinetic Monte Carlo simulations of organic photovoltaic devices: The treatment of electrostatic interactions," J. Chem. Phys. 132(9), 094705 (2010).

119 P. P. Ewald, "Die Berechnung optischer und elektrostatischer Gitterpotentiale," Ann. Phys. 369(3), 253-287 (1921).

${ }^{120}$ T. Darden, D. York, and L. Pedersen, "Particle mesh Ewald: An $N \cdot \log (N)$ method for Ewald sums in large systems," J. Chem. Phys. 98(12), 10089-10092 (1993).

${ }^{121}$ U. Essmann, L. Perera, M. L. Berkowitz, T. Darden, H. Lee, and L. G. Pedersen, "A smooth particle mesh Ewald method," J. Chem. Phys. 103(19), 8577-8593 (1995).

${ }^{122}$ W. R. Saunders, J. Grant, E. H. Müller, and I. Thompson, "Fast electrostatic solvers for kinetic Monte Carlo simulations," J. Comput. Phys. 410, 109379 (2020). 
${ }^{123} \mathrm{M}$. Pippig and F. Mercuri, "Efficient evaluation of Coulomb interactions in kinetic Monte Carlo simulations of charge transport," J. Chem. Phys. 152(16), 164102 (2020).

${ }^{124}$ T. Bereau, D. Andrienko, and K. Kremer, "Research update: Computational materials discovery in soft matter," APL Mater. 4(5), 053101 (2016).

${ }^{125}$ T. Bereau, D. Andrienko, and O. A. von Lilienfeld, "Transferable atomic multipole machine learning models for small organic molecules," J. Chem. Theory Comput. 11(7), 3225-3233 (2015).
${ }^{126}$ C. Scherer, R. Scheid, D. Andrienko, and T. Bereau, "Kernel-based machine learning for efficient simulations of molecular liquids," J. Chem. Theory Comput. 16(5), 3194-3204 (2020).

${ }^{127}$ S. Yin, Z. Shuai, and Y. Wang, "A quantitative structure-property relationship study of the glass transition temperature of OLED materials," J. Chem. Inf. Comput. Sci. 43(3), 970-977 (2003).

${ }^{128} \mathrm{~J}$. Xu and B. Chen, "Prediction of glass transition temperatures of OLED materials using topological indices," J. Mol. Model. 12(1), 24-33 (2005). 